\title{
Parkinsonism and transient bilateral ptosis in systemic lupus erythematosus
}

\author{
P. C. TEOH \\ M.B.(HK), M.Med.(Singapore), M.R.C.P.(UK), A.M. \\ A. T. Ng RICHARD \\ M.B., B.S.(Singapore) \\ P. K. WONG \\ M.B.(Malaya), F.R.C.P., F.R.C.P.(E), A.M.
}

\section{Department of Medicine, University of Singapore, Singapore}

\begin{abstract}
Summary
Many neurological abnormalities have been described in systemic lupus erythematosus (SLE), but transient bilateral ptosis and parkinsonism are rarely encountered. This paper describes a young Malay girl with SLE who develops psychosis, bilateral ptosis and parkinsonism during an exacerbation of her illness. These neurological features disappeared after adequate treatment with cyclophosphamide. Though the pathogenesis of these neurological abnormalities is not clearly known, it is likely that transient bilateral ptosis is due to myoneural dysfunction not unlike that of myasthenia gravis. As for parkinsonism, it can probably be explained on the basis of 'vasculitis' of the basal ganglia leading to microinfarcts and encephalomalacia.
\end{abstract}

SYSTEMIC lupus erythematosus (SLE) is a generalized condition affecting the connective tissues of many organs. Though it affects particularly the skin and joints, it can have many forms of presentations and sometimes the disorder of single system may dominate the picture. Symptoms referable to central nervous system are commonly found in SLE and can occasionally be the presenting features. In a recent large prospective study of SLE, Estes and Christian (1971) observed that central nervous system involvement was found in $59 \%$ of patients. The symptoms may vary from neurosis to psychosis. Convulsive seizures of any type can occur. In addition peripheral neuropathy, cranial nerve disorders, paralysis like hemiplegia, quadraplegia and paraplegia, chorea and myasthenia gravis have often been reported. In this paper, we report a case of SLE with two very unusual features: transient bilateral ptosis and parkinsonism.

\section{Case report}

S.B.A., a 12-year-old Malay girl, was first seen in August, 1970 with fever for 5 days. There was associated swelling on the left side of the neck. She also gave a history of puffiness of the eye-lids on and off for the last 6 months. Clinical examination revealed an intelligent girl in fairly good general condition. She was slightly anaemic and had a temperature of $102^{\circ} \mathrm{F}$. There was minimal oedema of the eye-lids and mild proptosis but no ptosis was observed. The left tonsillar lymph node was enlarged and tender. Her pulse rate was $140 / \mathrm{min}$ and B.P. $110 / 70 \mathrm{mmHg}$. Liver was palpated $2 \mathrm{~cm}$ below right costal margin and the spleen was just palpable. Her mental faculty was normal and there was no clinical evidence of neurological deficits.

Laboratory investigations showed that her haemoglobin was $9 \mathrm{~g} \%$ and her leucocyte count was $8300 / \mathrm{mm}^{3}$. Erythrocyte sedimentation rate (ESR) was $12 \mathrm{~mm} / \mathrm{hr}$. Lupus erythematosus (LE) cells and antinuclear factors (ANF) were found on many occasions. Serum globulin was $2.8 \mathrm{~g} \%$ and blood urea was normal. Urine contained 10 red blood cells, trace of albumin but no cast. A firm diagnosis of SLE was made. She was treated with prednisolone 40 $\mathrm{mg} /$ day. The response was good as evidenced by subsidence of fever and reduction in size of the enlarged lymph node over the next few days. The dosage of prednisolone was reduced over the next few weeks to $15 \mathrm{mg} /$ day. On discharge, her ESR was only $2 \mathrm{~mm} / \mathrm{hr}$ though her LE cells and ANF remained positive.

For the next 20 months, she was well while maintained on prednisolone $15 \mathrm{mg} /$ day except on two occasions she had recurrence of fever when her prednisolone was reduced to $5 \mathrm{mg} /$ day. On the twenty-first month, patient was readmitted because of abnormal behaviour, restlessness and wandering about aimlessly at night. Except for her prednisolone which she took faithfully $5 \mathrm{mg}$ three times a day, there was no history of taking any other drugs. On clinical examination she was found to be disorientated and very careless in her dress and look. She exhibited frank paranoid thought and behaviour and was obviously psychotic. Careful neurological examination revealed bilateral ptosis (Fig. 1) which was of sufficient degree for us to suspect her having myasthenia gravis though there was no other evidence to support it. Her gait was shuffling and her limbs were hypertonic showing cogwheel rigidity. Resting tremor of her hands was also noted. Glabella tap was repeatedly positive. It became 


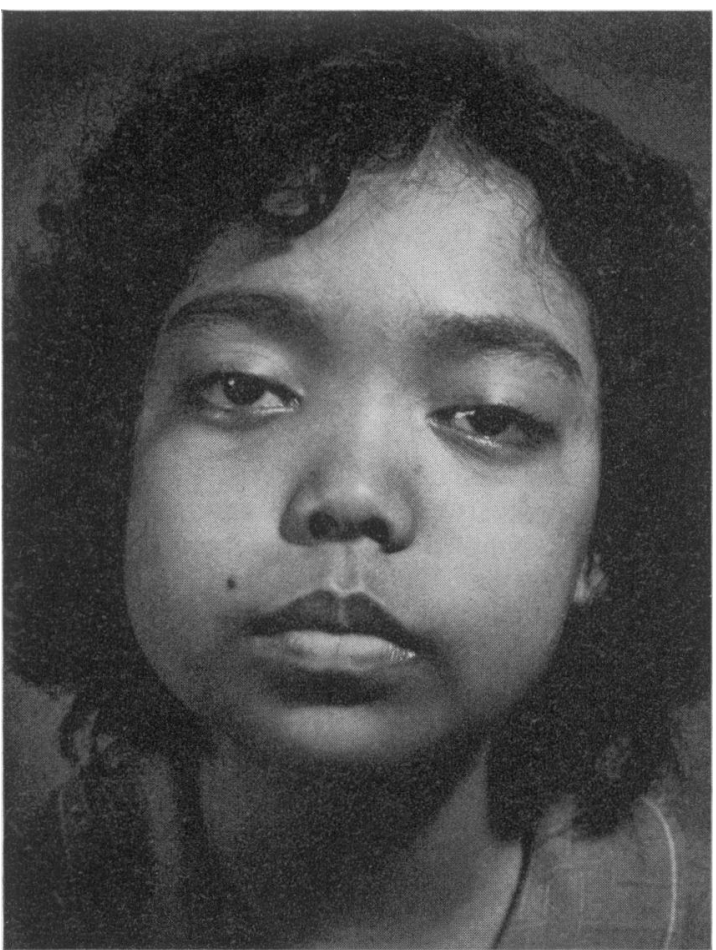

Fig. 1. Bilateral ptosis and expressionless facies.

obvious that she had many features of extrapyramidal disorders not unlike those of parkinsonism. Laboratory investigations showed an ESR of $2 \mathrm{~mm} / \mathrm{hr}$ but the LE cells and ANF were positive. Serum globulin was $3 \mathbf{g} \%$ with gamma globulin of $1.1 \mathrm{~g} \%$. Immunoglobulin study showed IgG of 2800 $\mathrm{mg} \%$, IgA of $280 \mathrm{mg} \%$ and IgM of $65 \mathrm{mg} \%$. Electroencephalogram and cerebrospinal fluid were normal.

As the psychosis came on after 20 months of prednisolone therapy the possibility of it being steroid-induced was considered. She was taken off her prednisolone, but no improvement was noted after 2 weeks. Therefore we considered at this stage that the psychosis, bilateral ptosis and parkinsonism were the result of the exacerbation of her SLE. Prednisolone was restarted at $60 \mathrm{mg} /$ day and about 1 week later oral cyclophosphamide $100 \mathrm{mg} /$ day was added. Improvement of her symptoms became noticeable only during the second week after cyclophosphamide was added. At that time prednisolone was gradually reduced in its dosage. One month later, features of parkinsonism and bilateral ptosis disappeared (Fig. 2). As for her psychosis, the improvement was slow but steady. It took about 2 months before we could convince ourselves that she had completely recovered. By then the patient was only on cyclophos-

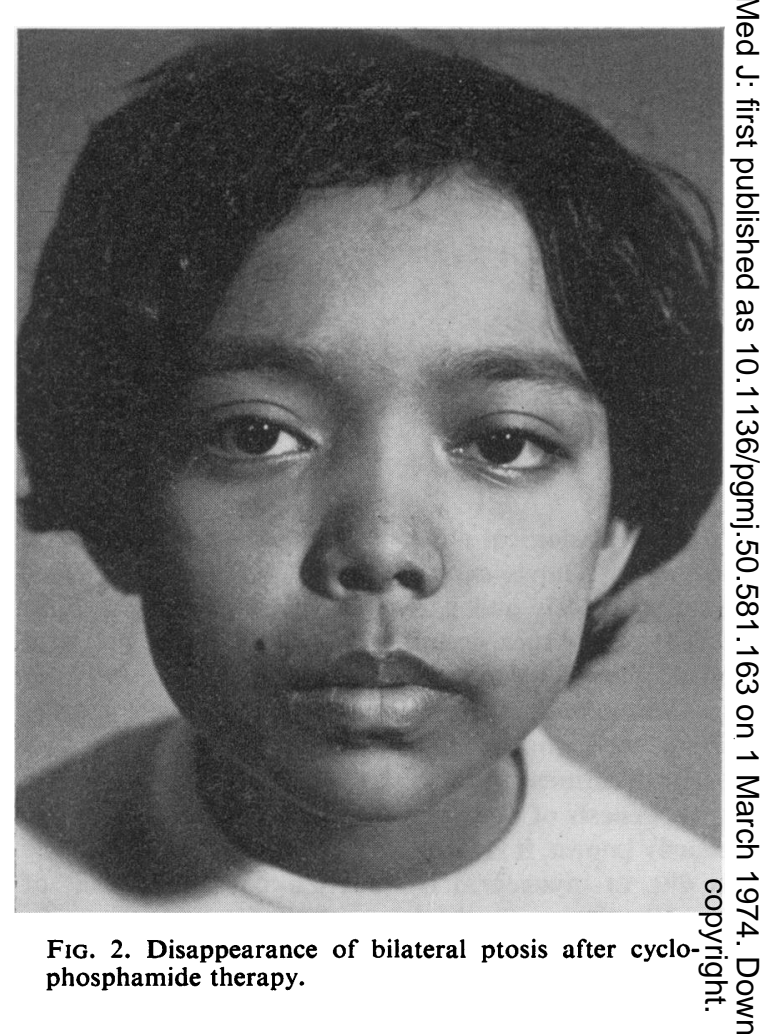

phamide and the side effect was obvious as her alopecia was marked and at one stage leucopenia severe. $\frac{\circ}{\square}$ She was taken off the cyclophosphamide temporarily $\varrho$ and was restarted at $50 \mathrm{mg} /$ day with lesser side $\overrightarrow{\overrightarrow{0}}$ effects.

\section{Discussion}

The diagnosis of systemic lupus erythematosus in 3 . this young girl is clearly established by the findings of $\frac{}{\circ}$ LE cells and positive antinuclear factor on many occasions in spite of the fact that ESR remains low. This therefore confirms the belief that it is not $₹$ necessary for all patients with systemic lupus 응 erythematosus to have elevated ESR as Dubois (1966) $>$ in his study of 463 patients found an elevation of ESR in only $84 \%$ of his cases.

Transient bilateral ptosis is a very unusual neuro-o logical feature of SLE. Neither Dubois and Tuffa- $N$ nelli (1964) or Clark and Bailey (1956) observed such స్ట a feature in their respective large series. However, 0 Lewis, Sinton and Knott (1954) noted one patiento had recurrent bilateral ptosis and Harvey et al. (1954) in their study of 138 patients with SLE found three? cases with transient bilateral ptosis. In one of them the diagnosis of myasthenia gravis was initially made but treatment with neostigmine did not improve the $\stackrel{\mathbb{Q}}{\stackrel{Q}{Q}}$ ptosis. The diagnosis was revised when the patient $\stackrel{\mathbb{Q}}{\stackrel{\mathrm{Q}}{\circ}}$ 
returned a few months later with complete disappearance of bilateral ptosis though she was not put on any specific treatment. In our patient we also initially suspected that she might have suffered from myasthenia gravis. However, her bilateral ptosis was only transient and disappeared 1 month after cyclophosphamide therapy. The cause of this transient bilateral ptosis is unknown and the anatomical level of the lesion is often in doubt. Probably it can be explained on the basis of myoneural dysfunction resembling myasthenia gravis (Harvey and Johns, 1962) and such cases often responded poorly to anticholinesterase therapy.

A variety of movement disorders have been described as unusual neurological manifestations of systemic lupus erythematosus. Among them are cerebellar ataxia and chorea which are by far the most widely reported disorders of movement (Rowe, 1963). Though tremor has also been reported, parkinsonism is almost an unknown neurological manifestation of systemic lupus erythematosus. Review of the studies made by Harvey et al. (1954), Clark and Bailey (1956), Dubois (1966), Tay (1971) and Estes and Christian (1971) does not describe such a feature. It was Seminario and Pessano in 1930 who first described four patients with systemic lupus erythematosus developed 'cigarette rolling' tremor with rigidity resembling paralysis agitans and Willoughby, Cardon and Rubritz in 1964 described one patient with cogwheel rigidity without tremor and at autopsy multiple areas of encephalomalacia were observed in basal ganglia. In 1966, O'Conner and Musher made brief mention that parkinsonianlike features were observed among their patients with SLE.

We have no doubt that our patient under study had clinical features of parkinsonism and they disappeared 1 month after treatment with cyclophosphamide. As we realized, parkinsonism could be induced by many drugs especially those of phenothiazines group which might be used by our patient for the treatment of her psychosis prior to her admission into the hospital, a very careful drug history was taken and there was no evidence to suggest that her parkinsonism was drug-induced. Though parkinsonism is an extremely uncommon neurological manifestation of systemic lupus erythematosus, there is no reason to believe that this cannot occur. Dubois (1966) was always impressed by the different presenting neurological features and the extreme variability of the central nervous system changes which might occur in SLE. He believed that no part of the central nervous system was immune from the attack. The etiological diagnosis of any neurological presentation depended upon proving the presence of other conditions which might produce similar neurological changes. In our patient reported above, the presence of SLE is proven and we are unable to elicit any other cause for her parkinsonism. We have ruled out drug, carbon monoxide and manganese poisoning as the cause. Idiopathic parkinsonism is almost unheard of in this age group. Postencephalitic parkinsonism is unlikely as there is no past history of encephalitis and rapid improvement of her parkinsonism features makes it most unlikely.

The basic pathological lesion of many neurological changes is the 'vasculitis' leading to haemorrhages, microinfarcts, perivascular fibrosis and encephalomalacia in the nervous system (Johnson, 1962; Johnson and Richardson, 1968). The autopsy finding of encephalomalacia in the basal ganglia in Willoughby's patient with cogwheel rigidity supports our belief that parkinsonism can be caused by 'vasculitis' in the corpus striatum particularly globus pallidus and substantia nigra. As chorea, another disorder of corpus striatum particularly the caudate necleus, is not too uncommonly associated with SLE (Paradise, 1960), it is therefore surprising to find that parkinsonism is so uncommon in patients with systemic lupus erythematosus.

\section{Acknowledgments}

We are grateful to Professor Sir Gordon Arthur Ransome, M.D., F.R.C.P., for his help in the study of this patient and preparation of this paper.

\section{References}

Clark, E.C. \& Bailey, A.A. (1956) Neurological and psychiatric signs associated with systemic lupus erythematosus. Journal of the American Medical Association, 160, 455.

Dubois, E.L. \& Tuffanelli, D.L. (1964) Clinical manifestations of systemic lupus erythematosus. Journal of the American Medical Association, 190, 104.

DuBoIs, E.L. (1966) Lupus Erythematosus. McGraw-Hill Book Company, New York.

Estes, D.C. \& Christian, C.L. (1971) The natural history of systemic lupus erythematosus by prospective analysis. Medicine, 50, 85.

Harvey, A.M., Shulman, L.E., Tumulty, P.A., Conley, C.L. \& SChOENRICH, E.H. (1954) Systemic lupus erythematosus: review of the literature and clinical analysis of 138 cases. Medicine (Baltimore), 38, 291.

HARVEY, A.M. \& JoHNS, R.J. (1962) Myasthenia gravis and the thymus. American Journal of Medicine, 32, 1.

JoHNSON, R.T. (1962) Neurological and neuropathological observations in lupus erythematosus. New England Journal of Medicine, 266, 895.

Johnson, R.T. \& Richardson, E.P. (1968) The neurological manifestations of systemic lupus erythematosus. Medicine, 47, 337.

LeWiS, B.I., Sinton, D.W. \& KNotT, J.R. (1954) Central nervous system involvement in disorders of collagen. Archives of Internal Medicine, 93, 315.

O'ConNer, J.F. \& Musher, D.M. (1966) Central nervous system involvement in systemic lupus erythematosus. Archives of Neurology, 14, 254.

Paradise, J.L. (1960) Sydenham's chorea without evidence of rheumatic fever. Report of its association with HenochSchoenlein syndrome, and with systemic lupus erythema- 
tosus, and review of literature. New England Journal of Medicine, 263, 625.

RowE, P.B. (1963) Disseminated lupus erythematosus with Sydenham's chorea: report of a case with a review of literature. Medical Journal of Australia, 2, 586.

Seminario, C. \& Pressano, J. (1930) Cuarto casos clinicos de lupus eritematoso agudo. Semaine médicale, 2, 721.
TAY, C.H. (1971) Neurological involvement in systemic lupus erythematosus. Singapore Medical Journal, 12, 18.

Willoughby, E.O., Cardon, L. \& Rubnitz, M.E. (1964) Clinopathologic conference; psychotic episodes, meningitis and chest pain. Postgraduate Medical Journal, 35, 318.

Postgraduate Medical Journal (March 1974) 50, 166-171.

\title{
Oxymetholone in aplastic anaemia
}

\author{
M. A. MIR \\ M.B., B.S., D.C.H., M.R.C.P. \\ I. W. Delamore \\ M.B., Ph.D., F.R.C.P.(Ed.), M.R.C.PATH. \\ University Department of Clinical Haematology, Manchester Royal Infirmary, \\ Oxford Road, Manchester, M13 9WL
}

\begin{abstract}
Summary
Thirty-one patients with aplastic anaemia were treated with oxymetholone. Twenty-eight patients lived beyond 2 months and of these eleven patients showed clinical and haematological improvement. Ten of these had some active erythropoietic areas as shown by bone marrow biopsies and by the presence of an elevated reticulocyte count in the peripheral blood. Two patients who responded in spite of a markedly hypoplastic marrow and reticulocytopenia became oxymetholone dependent and relapsed when the drug was withdrawn. They failed to respond a second time when oxymetholone was re-introduced. Side effects were frequent. These were mostly tolerable and reversible on reduction of the dose but three patients died of acute myeloblastic leukaemia and in three others the drug had to be withdrawn.
\end{abstract}

DURING recent years androgenic hormones have been used extensively in aplastic anaemia (Shahidi and Diamond, 1961; Sanchez-Medal et al., 1964; Reynafarje and Faura, 1967; Daiber et al., 1970). Promising reports have appeared in favour of oxymetholone which is an oral androgen. SanchezMedal et al. (1969) claimed a remission rate of $70 \%$ in patients with aplastic anaemia who lived longer than 2 months and thus received an adequate trial with oxymetholone. Similar results have been reported by Allen et al. (1968) and by Silink and Firkin (1968).

We present here our experience with oxymetholone on thirty-one patients with aplastic anaemia.
Patients and methods

Thirty-one patients were treated. All had pancyto penia and a marrow with reduced cellularity at some stage on serial trephine biopsies. In nine patients aplastic anaemia was considered to be drug-induced (Table 1). All other causes of anaemia were excluded by a careful clinical assessment and by haematological investigations. Patients with primary disease affecting the bone marrow by infiltration or by replacement of active haemopoietic tissue were excluded.

Haematological investigation and ferrokinetic studies were performed by standard methods (Dacie and Lewis, 1968). All patients had a sternal marrow aspiration and a trephine biopsy on the iliac crest. Both these procedures were repeated on seventeen patients to define the extent of aplasia and to assess the response to therapy. Sixteen patients died and autopsies were performed on nine. In these patients sections from the pelvis and from the vertebral column were examined histologically to get an overall picture of the marrow cellularity. Marrow cellularity was divided into the following sub-headings according to the observations made on the trephine sections:

(a) Marked hypoplasia. The trephine sections showed an occasional collection of lymphocytes or reticulum cells among the fatty cells. There was a $\stackrel{?}{+}$ severe depression of the erythro-myeloid elements and a complete absence of megakaryocytes.

(b) Moderate hypoplasia. A marked reduction of the overall cellularity but an occasional island of 